East African Medical Journal Vol. 84 No. 9 September 2007

ZINC FOR DIARRHOEA MANAGEMENT IN SUB-SAHARAN AFRICA: A REVIEW

C.L. Fischer Walker, PhD, Johns Hopkins Bloomberg School of Public Health, 615 North Wolfe St., Room 5543, Baltimore, MD 21205, S. Aboubaker, World Health Organization, Department of Child and Adolescent Health, Geneva, Switzerland, R. Van de Weerdt, UNICEF, Health Section Programme Division, New York, NY and R.E. Black, Johns Hopkins Bloomberg School of Public Health, 615 North Wolfe St., Room 6527, Baltimore, MD 21205

Request for reprints to: Dr. C.L. Fischer Walker, Johns Hopkins Bloomberg School of Public Health, 615 North Wolfe St., Room 5543, Baltimore, MD 21205

\title{
ZINC FOR DIARRHOEA MANAGEMENT IN SUB-SAHARAN AFRICA: A REVIEW
}

\author{
C.L. FISCHER WALKER, S. ABOLBAKER, R. VAN DE WEERDT and R.E. BLACK
}

\begin{abstract}
Objectives: To review the evidence supporting the inclusion of zinc for diarrhoea management specifically in sub-Saharan Africa where diarrhoea remains a leading cause of morbidity and mortality.

Data sources: We searched PubMed for studies assessing the efficacy and effectiveness of zinc for the treatment and prevention of common childhood morbidities.

Study selection: We included only studies conducted in sub-Saharan Africa.

Data synthesis: Details of studies conducted in sub-Saharan Africa are presented in the context of the global evidence supporting the use of zinc for diarrhoea management.

Conclusions: There is a significant body of evidence to support the use of zinc for diarrhoea management in sub-Saharan Africa. The accelerated introduction of zinc into routine communitybased diarrhoea treatment is critical for the reduction of diarrhoea morbidity and mortality.
\end{abstract}

\section{INTRODUCTION}

Diarrhoea remains a leading cause of morbidity and mortality among young children around the world (1). Though diarrhoea mortality rates have declined since the advent of Oral Rehydration Solution (ORS) in the early 1980s, incidence rates have not improved thus there is still a great need for improved diarrhoea management $(2,3)$. For more than 25 years, the only drug recommended by WHO and UNICEF for the prevention and treatment of dehydration was the single formulation of ORS. Caregivers continued to seek additional relief for sick children and often turned to inappropriate antibiotics or dangerous anti-diarrhoeals. For many years there were no safe and effective adjunct therapies for diarrhoea except in the cases of cholera and dysentery where antibiotic use is justified. In May 2004, following results of several studies, WHO and UNICEF released a joint statement recommending the use of a new lower osmolarity ORS and the addition of zinc for 10-14 days for diarrhoea management (4).

In sub-Saharan Africa, diarrhoea remains an important cause of morbidity and mortality in children under five years of age. Improving diarrhoea management and increasing access to treatment are priority actions needed in countries. Many countries in this region have begun to introduce and implement the new recommendations made by WHO and UNICEF. They have revised their national policies and standards of treatment. Some countries are also producing the new lower osmolarity ORS locally adhering to the WHO guidelines for the production of low osmolarity ORS. Though this change has taken time it is proving to be a relatively simple change for Ministries of Health 
because using a lower osmolarity ORS does not require behaviour change and the new formulation is slightly less expensive than the original. Those countries who order ORS from UNICEF receive the new low osmolarity ORS as this is the only formulation now available for restocking.

For many countries in sub-Saharan Africa adding zinc for diarrhoea into routine child health services has proven to be more challenging. There are a number of health system explanations for this including already overstretched national budgets and competing priorities due to a high burden of disease attributable to HIV/AIDS, tuberculosis and malaria. In addition, countries have relatively little experience with the use of zinc for diarrhoea management. Thus, zinc is still new in national and regional academic and paediatric circles. Though the scientific evidence supporting zinc for diarrhoea is strong, the majority of the research was conducted in South Asia. Questions have been raised with regards to the generalisability of this extensive body of evidence to the African context.

The aim of this paper is to assist policy makers, donors, and public health practitioners to better understand the need for zinc for diarrhoea management specifically in the African setting by describing the burden of diarrhoeal disease and risk of zinc deficiency among African children, and the global evidence supporting zinc for diarrhoea management. We searched PubMed using zinc
AND diarrhoea, zinc AND infectious disease, zinc AND growth, and zinc AND pneumonia. For the purposes of this review we limited the data presented to those studies conducted in sub-Saharan Africa.

\section{DIARRHOEA MORBIDITY AND MORTALITY AMONG CHILDREN UNDER FIVE}

For most of the developing world, including sub-Saharan African countries, the incidence of diarrhoeal disease has not declined over the past 50 years. In a comparison of longitudinal studies, where active morbidity surveillance was conducted for more than one year between 1992 and 2000, with those conducted from 1950 through the end of the 1980s, there were no differences in diarrhoea morbidity rates (2). Children under five years of age experienced on average 3.2 episodes of diarrhoea per year with the highest rates (median $=4.8$ episodes per year) among infants aged 6-11 months. The review included five studies conducted in subSaharan Africa (Figure 1) (5-9). The data suggest that diarrhoea rates in African children are at least as high as the global medians. And though other diseases such as malaria and HIV/AIDS compete for the attention of policy makers, diarrhoea remains one of the leading causes of childhood morbidity and mortality. More effort and attention is needed for improved diarrhoea management.

Figure 1

Incidence of diarrhoea among children under five in sub-Saharan Africa

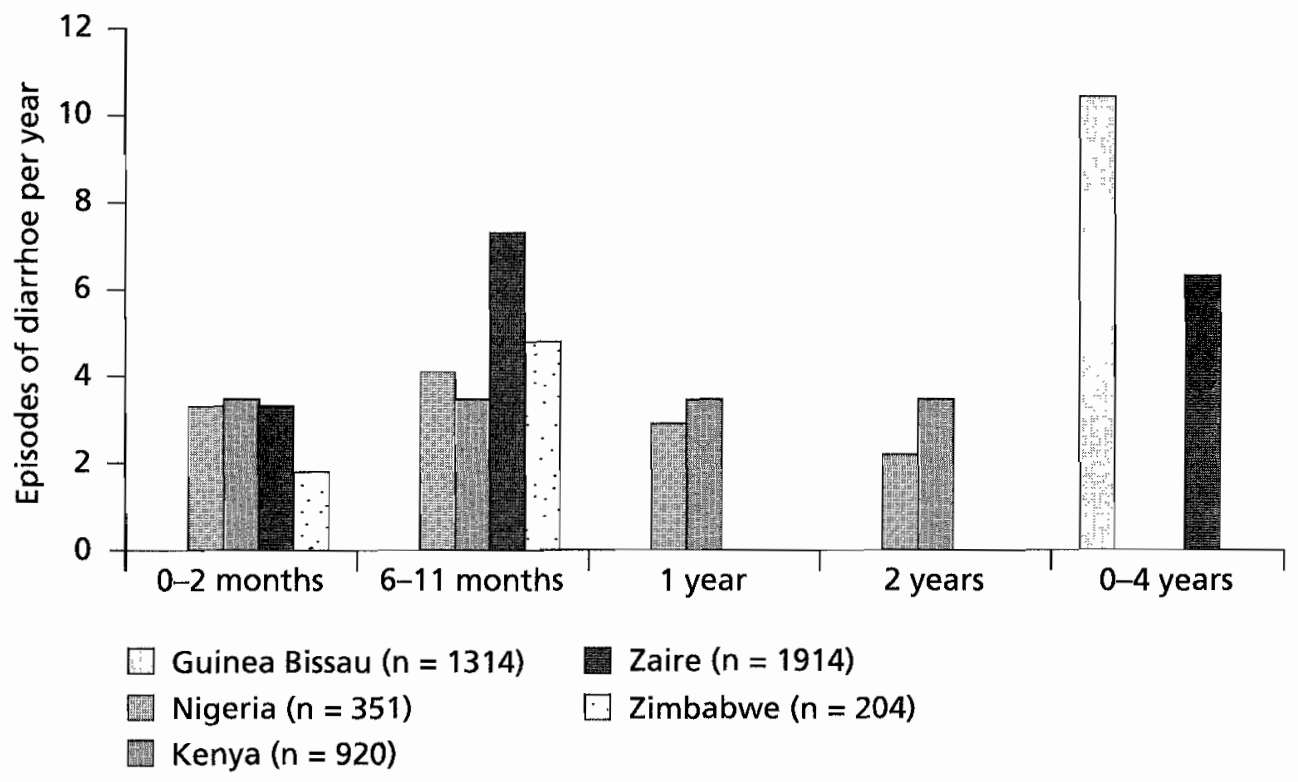


The 4th Millennium Development Goal (MDG) is to reduce mortality among children under five by two thirds. To ensure that this goal is reached, it is critical that policy makers at all levels recognise the leading causes of death. In sub-Saharan Africa, diarrhoea accounts for $16 \%$ of child deaths which is only slightly less than malaria (18\%) and far greater than AIDS (6\%) (Figure 2) (10). Though this varies by country (Figure 3), with rates less than $2 \%$ in South Africa, Botswana, and Mauritius and 20\% in Sierra Leone and Niger, policy makers must recognise that improving the outcome of all diarrhoea episodes will be crucial if under five mortality is to be cut by two-thirds in Africa.
Figure 2

Causes of death among children under five in Africa

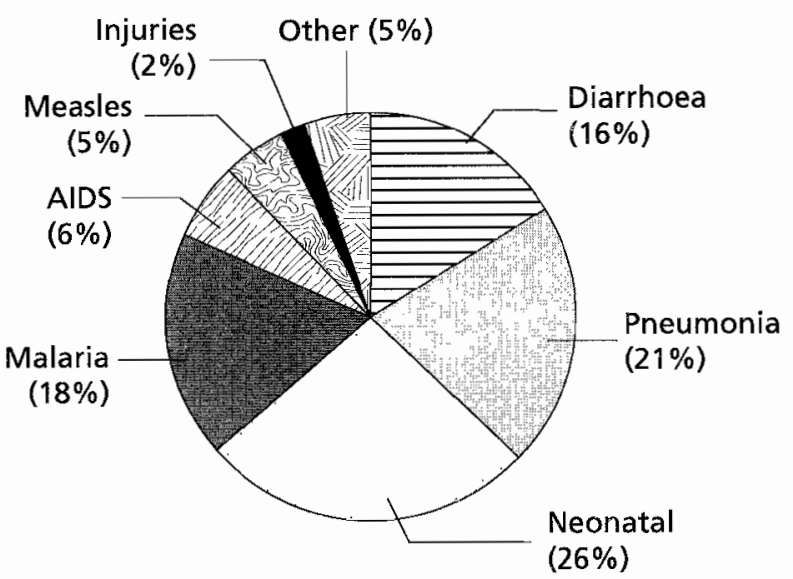

Figure 3

Percent of deaths attributed to diarrhoea by country

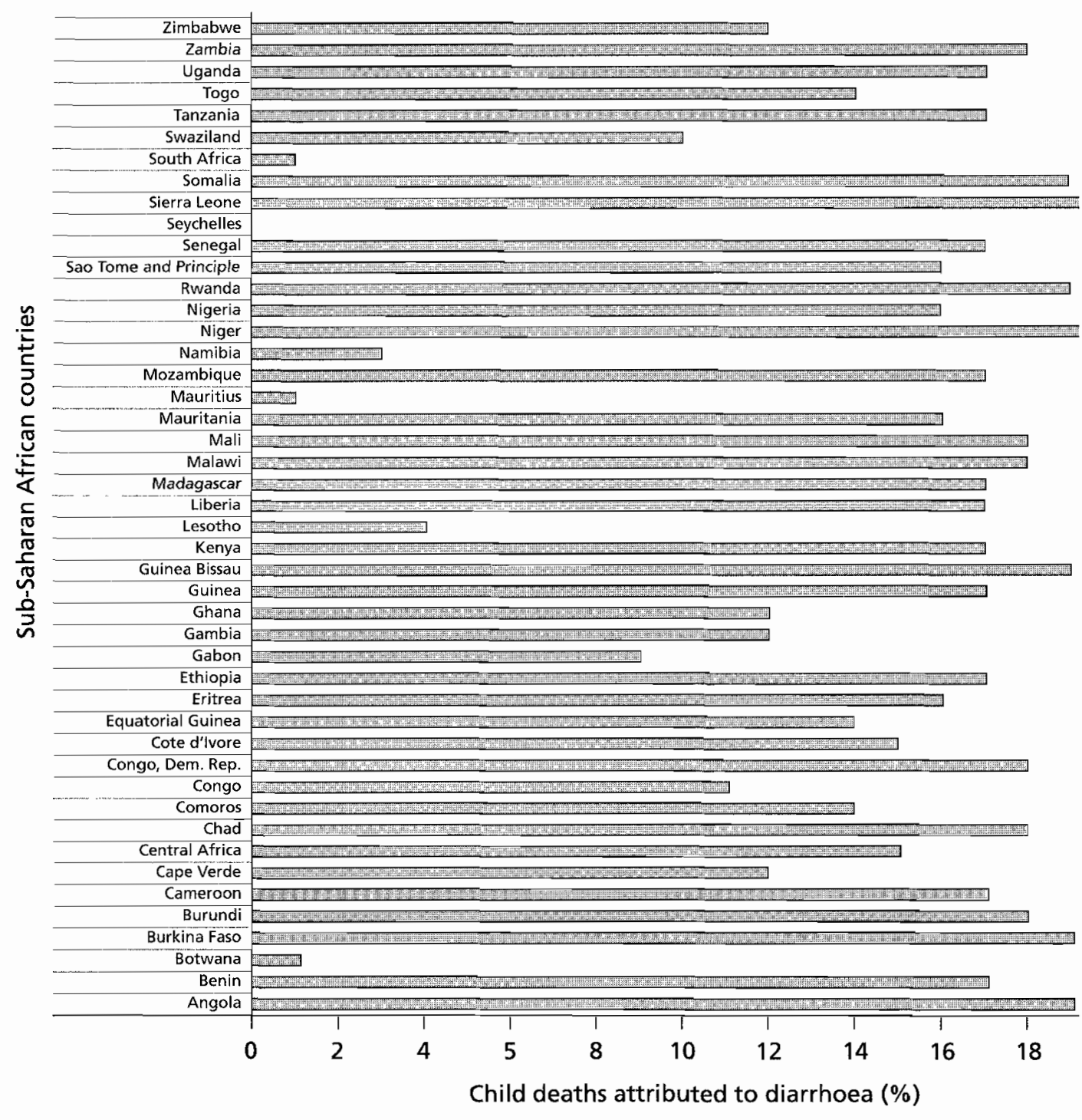




\section{RISK OF ZINC DEFICIENCY}

Human zinc deficiency was first observed by A.S. Prasad in the early 1960s. He recognised nutritional dwarfism among adolescent boys in Iran and Egypt which was then defined as severe zinc deficiency upon serum zinc analysis (11). Severe zinc deficiency is easy to recognise; common clinical features include severe growth retardation, delayed sexual maturation, and development of orificial and acral dermatitis (12). Mild to moderate zinc deficiency is more difficult to diagnose because the signs and symptoms, such as an increased susceptibility to infections and reduced growth rate, are also those associated with generalised malnutrition and frequent common childhood illnesses (12).

It is estimated that global zinc deficiency is responsible for the loss of 28 million Disability Adjusted Life Years (DALYs) including 789,000 child deaths each year (13). In many developing countries, children under five are at an increased risk for zinc deficiency because their diets are low in both total zinc and absorbable zinc (14). Children do not receive adequate quantities of animal products which have high zinc concentrations and their diets are often high in phytates (typically found in cassava, maize, and wheat products) which inhibit the absorption of zinc. Though the feeding habits of young children can help predict zinc deficiency in this population, stunting rates can also be used as an indicator for the risk of zinc deficiency (15). It is now recommended that the risk of zinc deficiency be estimated by combining the percent of the population at risk of inadequate zinc intake based on food balance sheets with the prevalence of stunting among children under five years old (15). Based on these data children living in sub-Saharan Africa are either at intermediate or high risk for zinc deficiency (Figure 4).

Dietary studies of feeding practices of young children in sub-Saharan Africa provide additional evidence that because of poor diets young children are at particular risk for zinc deficiency. The diets of 179 breastfed Malawian infants up to 24 months of age were assessed in a cross-sectional survey. In this survey all infants over four months of age were receiving complementary food which was predominately maize flour porridge for young children progressing to family foods as the child aged (16). For each age group the overall zinc intake was too low to meet the daily needs of the child given the low bioavailability of the zinc consumed. Given these dietary data it is not surprising that by the age of 18-24 months $46 \%$ of children were stunted. Mamabolo and colleagues assessed the dietary intake of 219 South African infants from birth to 12 months of age (17). In this population $85 \%$ of infants were still breastfed at nine months of age, yet by three months of age $83.5 \%$ of them were receiving at least one complementary food. The most common complementary foods in this age group were maize meal and sorghum, both prepared in a porridge and low in bioavailable zinc. At birth, infants in this

Figure 4

National risk of zinc deficiency based on prevalence of stunting among children under five and absorbable zinc content of the food supply

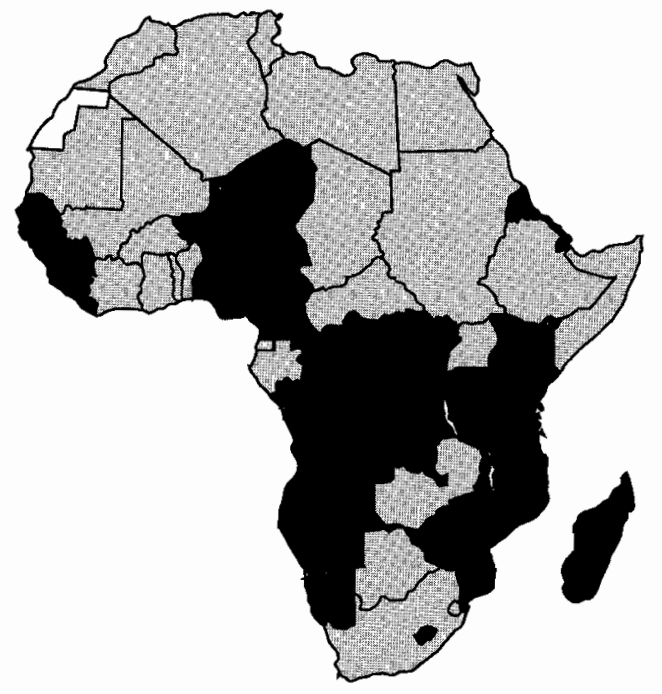


cohort had low height for age $Z$ scores (mean $=-0.51$ HAZ) which continued to worsen throughout the first year of life resulting in 38\% of infants stunted by 12 months of age (mean $=-1.38 \mathrm{HAZ}$ ). Mamiro and colleagues observed that among Tanzanian children 3-23 monthsof age, carbohydrates accounted for 69\% of the energy intake and protein sources, which are high in zinc, accounted for only $12 \%$ (18). In a 24 hour dietary recall, maize porridge was the most frequently reported complementary food. Mothers reported giving it at all ages with increasing viscosity as the child got older. The diets of these young children did not provide for the iron needs of infants in any age group. Daily intake provided only $15 \%$ of the iron needs for infants 6-8 monthsof age, $20 \%$ for infants 9-11 months of age, and 27\% for infants 12-23 months of age. Though the zinc content was not assessed in this study, it is possible to use these data of inadequate dietary iron to suggest there was also inadequate zinc intake. This assumption is reasonable because iron is found in the same food sources as zinc and like zinc has diminished bioavailability as the proportion of phytates in the meal increases. These dietary studies support estimates made from national level food balance sheets and further illustrate that zinc deficiency is a widespread problem affecting disadvantaged African children. Because zinc deficiency increases the child's risk for diarrhoea and pneumonia in addition to negatively impacting growth, interventions to correct zinc deficiency should be top child health priorities.

There have been 29 randomized controlled trials of long term, daily or weekly, zinc supplementation powered to detect a significant effect of zinc on the reduction of morbidity and/or mortality. Twentyfive of the 29 trials demonstrated one or more benefits of zinc on infectious disease morbidity or mortality. The effect of zinc on growth was studied in 28 randomized controlled studies (some assessed only growth and others also assessed morbidity and morality and are also included in the total above). Zinc had a positive effect on growth in 21 of the 28 studies.
Six trials of daily or weekly on-going zinc supplementation vs. a placebo or control have been conducted in sub-Saharan Africa (19-24). All of these studies demonstrated a positive effect of zinc on one or more indicator(s) of morbidity, mortality, and / or growth (Table 1). In the recently completed largescale, community-based randomized trial of 42,546 children on the Zanzibar island of Pemba there was an $18 \%$ reduction in all-cause mortality among zincsupplemented children 12 months of age and older compared to those who did not receive zinc (23). The effect was stronger among boys than girls and represented a decrease in infectious disease mortality including diarrhoea, pneumonia, and malaria. HIVpositive children in South Africa were randomized to receive zinc or placebo for six months to evaluate the safety of supplementation in this population (20). Zinc-supplemented children gained more weight and had fewer infections. There were 2 deaths among zinc supplemented compared to 7 who received the placebo $(p=0.1)$. In a study conducted in Burkina Faso zinc-supplemented children experienced fewer days of diarrhoea compared to children who received placebo (22). This study also observed a trend in mortality reduction. In Ethiopia, Umeta and colleagues randomized both stunted and non-stunted children to receive zincor placebo and reported fewer new infections among stunted children who received zinc compared to those who received placebo (24). Zinc also positively affected growth in this population; this effect was more pronounced among infants who were stunted at baseline. Among Ugandan children, 33-89 months of age, zinchad a small non-significant affect on infectious disease weekly incidence (1.36 vs. 1.82 episodes per week, $p=0.06)$ and increased arm circumference after six months of supplementation $(p<0.05)$ (21). In The Gambia, zinc was provided twice weekly instead of daily (19). After 1 month of supplementation, children who received zinc had fewer visits to a health care provider for malaria compared to children who received placebo. There was also a small positive benefit of zinc on arm circumference and weight gain among children attending schools of mid-socioeconomic class. 


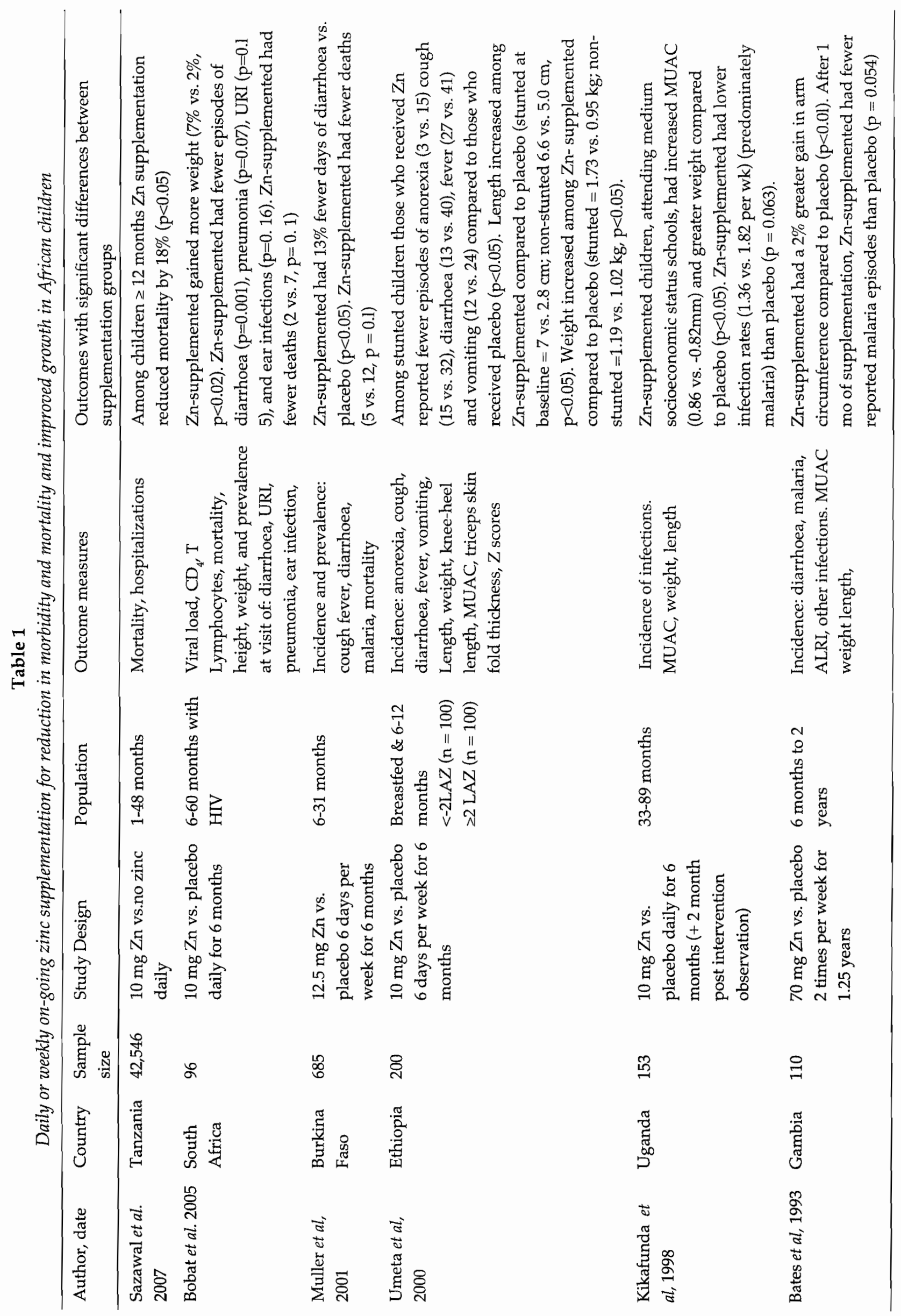




\section{EVIDENCE SUPPORTING ZINC IN DIARRHOEA MANAGEMENT}

Globally there have been 15 randomized placebocontrolled trials evaluating the effect of zinc as an adjunct therapy for acute diarrhoea and five randomized placebo-controlled trials evaluating zinc for persistent diarrhoea. Among children with acute diarrhoea, zinc has been shown to decrease the duration of the diarrhoea episode by $15 \%$ and significantly reduce stool output $(25,26)$. In children with persistent diarrhoea, zinc has been shown to decrease the duration of the episode by $24 \%$ and reduce the risk of treatment failure or death by $42 \%$ (26). Fourteen days of zinc for diarrhoea has also been to shown to decrease the incidence of diarrhoea and pneumonia in the 2-3 months following the diarrhoea episode (27). In addition to these important benefits on the diarrhoea episode and prevention of morbidity, zinc for diarrhoea has also been shown to decrease mortality. In one large community-randomized trial of more than 11,000 child years of observation over two years, children living in villages where zinc for diarrhoea was available had a $51 \%$ lower risk of mortality as compared to children living in the control clusters (25). The body of scientific literature supporting zinc for diarrhoea management is extensive. It is for this reason that WHO and UNICEF formulated the global recommendations and issued joint statements to support countries translate the evidence into action (4). Zinc has also been incorporated into the WHO Essential Medicines List thus making it possible for zinc product to be stocked in the UNICEF warehouse.

There are attempts to strengthen the evidence base regarding zinc for diarrhoea in the African region. One study that specifically assessed the effect of zinc for diarrhoea among infants 1-5 months of age was conducted in Ethiopia, India, and Pakistan (28). One thousand one hundred and ten infants were randomized (187 in Ethiopia) to receive 10 mg zinc or placebo daily for 14 days as an adjunct treatment for diarrhoea. Infants in this study did not benefit from zinc for diarrhoea treatment but experienced no adverse events; this was observed overall and when stratified by study site. Though these results differ greatly from the benefit of zinc on diarrhoea that is well documented among older children, they are consistent with the only other published study of infants less than six months of age, conducted in Bangladesh, which also observed no effect of zinc on diarrhoea (29). In a multicountry study assessing the acceptability of zinc for diarrhoea, 248 Ethiopian children were among the 2002 children randomized to receive zinc in addition to ORS or ORS alone for diarrhoea treatment (30). The results of this five country study demonstrated that zinc for diarrhoea does not displace ORS use and has the ability to displace the inappropriate use of antibiotics. The positive effects of zinc on diarrhoea treatment behaviour were similar across four of the countries which included Ethiopia. A large scale cluster randomised zinc implementation study is currently underway in Mali. This study is investigating implementation strategies, behaviour change message development, and cost effectiveness. Results from the pilot stage of this study suggest that acceptability and compliance will be high in the African context ( $89 \%$ of children received at least 10 of the 14 recommended days of zinc) (31).

Although the use of zinc for routine management of diarrhoeal diseases has not until recently been part of Ministry of Health national policies, zinc has been used widely in the African region particularly in emergency situations. The low cost and effectiveness of zinc for diarrhoea treatment has contributed to its use in refugee camps such as the many managed by the International Rescue Committee (IRC). Thousands of children have been treated in camps in the Democratic Republic of Congo, Sierra Leone, Sudan, Chad, and Rwanda using zinc as part of the routine management of diarrhoea. In a recent cholera outbreak in Botswana, zinc was included as part of the crisis management strategy to decrease the duration and severity of the diarrhoea episode.

Zinc is now available from the UNICEF supply division, thus it is expected that the number of national governments and NGOs purchasing zinc for use as part of improved diarrhoea management for all children will continue to grow.

\section{CONCLUSION}

Diarrhoea remains a leading cause of death among children under five in Africa. To reduce childhood mortality by two-thirds, and achieve the MDGs, improved diarrhoea management is critical. Using current knowledge and political will, the world has the capacity to make this happen. Adding zinc to 
diarrhoea management has widespread scientific backing as a result of numerous studies including children from around the world. It is proving to be a simple addition to diarrhoea management strategies globally. Zinc reduces the duration and severity of the diarrhoea episode and is an inexpensive addition to ORS for the management of childhood with diarrhoea episodes; thus it should be considered a top priority for improving child health. Zinc deficiency is widespread among African children and studies of on-going daily supplementation have demonstrated a positive response to zinc supplementation with regard to growth and resistance to infectious diseases including diarrhoea, pneumonia, and malaria. Though additional research is underway to identify the best programmatic strategy to increase the daily intake of zinc in young children on a more on-going basis, zinc for diarrhoea is a simple and effective way to provide a life saving treatment and the short term benefits of added zinc, namely improved protection from infections for 2-3 months following short-course supplementation. There is a need for an accelerated roll out of zinc in management of diarrhoea. There is increasing commitment to saving the lives of millions of children through the addition of zinc to national diarrhoea management strategies worldwide.

\section{ACKNOWLEDGEMENTS}

This paper was funded by Bill and Melinda Gates Foundation through the work of Global Zinc Task Force. The authors alone are responsible for the views expressed in this publication and they do not necessarily represent the decisions or the stated policy of the World Health Organisation or UNICEF.

\section{REFERENCES}

1. Bryce J., Boschi-Pinto C., Shibuya K. and Black R.E. WHO estimates of the causes of death in children. Lancet. 2005; 365: 1147-1152.

2. Kosek M., Bern C. and Guerrant R.L. The global burden of diarrhoeal disease, as estimated from studies published between 1992 and 2000. Bull. WHO. 2003; 81: 197-204.

3. Victora C.O., Bryce J., Fontaine O. and Monasch R. Reducing deaths from diarrhoea through oral rehydration therapy. Bull. WHO. 2000; 78: 1246-1255.
4. WHO, UNICEF. WHO-UNICEF Joint statement on the clinical management of acute diarrhoea. World Health Assembly. Geneva, 2004.

5. Manunébo M.N., Haggerty P.A., Kalengaie M., Ashworth A. and Kirkwood B.R. Influence of demographic, socioeconomic and environmental variables on childhood diarrhoea in a rural area of Zaire. J. Trop. Med. Hyg. 1994; 97: 31-38.

6. Mirsa N.M., Caulfield L.E., Black R.E. and Macharia W.M. Risk factors for diarrhoeal duration. Amer. J. Epidemiol. 1997; 146: 776-785.

7. Molbak K., Jensen H., Ingholt L. and Aaby P. Risk factors for diarrhoeal disease incidence in early childhood: A community cohort study from GuineaBissau. Amer. J. Epidemiol. 1997; 146: 273-282.

8. Moy R.J., Booth I.W., Choto R.G. and McNeish A.S. Recurrent and persistent diarrhoea in a rural Zimbabwean community: a prospective study. J. Trop. Pediatr. 1991; 37: 293-299.

9. Oni G.A., Schumann D.A. and Oke E.A. Diarrhoeal disease morbidity, risk factors and treatments in a low socioeconomic area of Ilorin, Kwara State, Nigeria J. Diarrhoeal Dis. Res. 1991; 9: 250-257.

10. Child Health Epidemiobogical Reference Group. Causes of under-five deaths. In: UNICEF/DPP/SIS, ed., 2006.

11. Prasad A.S., Miale A., Farid Z., Sanstead H.H., Schubert A.R. and Darby W.J. Biochemical studies on dwarfism, hypogonadism and anemia. Arch. Int. Med. 1963; 111: 407-428.

12. WHO. Zinc. Trace elements in human nutrition and health. Geneva: WHO, 1996.

13. Caulfield L.E. and Black R.E. Zinc deficiency. In: Ezzati M., Lopez A.D., Rodgers A. and Murray C.J.L. eds. Comparative quantification of health risks: Global and regional burden of disease attributable to selected major risk factors. Geneva: World Health Organization, 2004; 257-279.

14. Brown K.H., Rivera J.A., Bhutta Z., et al. Assessment of the risk of zinc deficiency in populations. Food $\mathcal{E}$ Nutr. Bull. 2004; 25: S130-S162.

15. Fischer Walker C.L. and Black R.E. Functional indicators for assessing zinc deficiency. Food \& Nutr. Bull. (In Press).

16. Hotz C. and Gibson R.S. Complementary feeding practices and dietary intakes from complementary foods amongst weanlings in rural Malawi. Eur.J. Clin. Nutr. 2001; 55: 841-849.

17. Mamabolo R.L., Alberts M., Mbenyane G.X., et al. Feeding practices and growth of infants from birth to 12 
months in the central region of the Limpopo Province of South Africa. Nutrition. 2004; 20: 327-333.

18. Mamiro P.S., Kobsteren P., Roberfroid D., Tatala S., Opsomer A.S. and Van Camp J.H. Feeding practices and factors contributing to wasting, stunting, and irondeficiency anaemia among 3-23-month old children in Kibosa district, rural Tanzania. J. Hlth. Popul. Nutr. 2005; 23: 222-230.

19. Bates C.J., Evans P.H., Dardenne M., et al. A trial of zinc supplementation in young rural Gambian children. Brit. J. Nutr. 1993; 69: 243-255.

20. Bobat R., Coovadia H., Stephen C., et al. Safety and efficacy of zinc supplementation for children with HIV-1 infection in South Africa: A randomised double-blind placebo-controlled trial. Lancet. 2005; 366: 1862-1867.

21. Kikafunda J.K., Walker A.F., Allan E.F. and Tumwine J.K. Effect of zinc supplementation on growth and body composition of Ugandan preschool children: A randomized, controlled, intervention trial. Amer. J. Clin. Nutr. 1998; 68: 1261-1266.

22. Muller O., Becher H., van Zweeden A.B., et al. Effect of zinc supplementation on malaria and other causes of morbidity in west African children: Randomised double blind placebo controlled trial. Brit. Med. J. 2001; 322: 1567.

23. Sazawal S., Black R.E., Ramsan M., et al. Effects of zinc supplementation or mortality in children 1-48 months of age: A community-based randomized placebocontrolled trial. Lancet. 2007; 369: 927-934.

24. Umeta M., West C.E., Haidar J., Deurenberg P. and Hautvast J.G. Zinc supplementation and stunted infants in Ethiopia: A randomised controlled trial. Lancet. 2000; 355: 2021-2026.

25. Baqui A.H., Black R.E., El Arifeen S., et al. Effect of zinc supplementation started during diarrhoea on morbidity and mortality in Bangladeshi children: Community randomised trial. Brit. Med. J. 2002; 325: 1059.

26. Zinc Investigators' Collaborative Group. Therapeutic effects of oral zinc in acute and persistent diarrhoea in children in developing countries: Pooled analysis of randomized controlled trials. Amer. J. Clin. Nutr. 2000; 72: 1516-1522.

27. Zinc Investigators' Collaborative Group. Prevention of diarrhoea and pneumonia by zinc supplementation in children in developing countries: Pooled analysis of randomized controlled trials. J. Pediatr. 1999; 135: 689-697.

28. Fischer Walker C.L., Bhutta Z., Bhandari N., et al. Zinc supplementation for the treatment of diarrhoea in infants in India, Pakistan, and Ethiopia. J. Pediatr. Gastroenterol. Nutr. 2006; 43: 357-363.

29. Brooks W.A., Santosham M., Roy S.K., et al. Efficacy of zinc in young infants with acute watery diarrhoea. Amer. J. Clin. Nutr. 2005; 82: 605-610.

30. INCLEN. Zinc supplementation in acute diarrhoea is acceptable, does not interfere with oral rehydration, and reduces the use of other medications: a randomized trial in five countries. J. Paed. Gastr. Nutr. 2006; 42: 300-305.

31. Winch P.J., Gilroy K.E., Doumbia S., et al. Prescription and administration of a 14-day regimen of zinc treatment for childhood diarrhoea in Mali. Amer. J. Trop. Med. Hyg. 2006; 74: 880-883. 